\title{
Les Modalites D'applications Des Regles Du Droit Economique Congolais Dans La Pratique Du Commerce En Ligne
}

\author{
Menga Yaosuwa JR*, Imani Gaya \\ Assistants to the Faculty of Law, University of BUNIA, Congo
}

DOI: $10.36348 /$ sijll.2020.v03i03.003

| Received: 02.03.2020 | Accepted: 12.03.2020 | Published: 18.03.2020

*Corresponding author: Menga Yaosuwa JR

\section{Abstract}

Il est une évidence opportune aujourd'hui de clamer la nécessité d'aborder la consolidation et l'enrichissement des dispositions encadrant la pratique "normale" du commerce en République Démocratique du Congo avant d'envisager les prétentions d'harmonisation et d'adaptation aux nouvelles technologies. Il ressort de l'irruption de l'internet, une réalité de développement sans précédent des transactions modifiant considérablement la perspective traditionnelle du commerce classique en le plongeant dans un mode électronique inhabituel, virtuel et ouvert. Internet ouvert à tous les coins et recoins du monde se retrouve soumis aux lois quels qu'en soient l'ordre juridique. De ce fait, nous conviendrons qu'internet n'est pas à l'ombre du droit d'autant plus qu'il est soumis à une pléthore des règles juridiques parfois distinctes mais, qui de concert avec certaines conventions sont promptes à circonscrire l'univers virtuel qu'il incarne. D'où il s'avère impérieux que les Etats du monde s'avisent à harmoniser leurs législations respectives pour une meilleure règlementation du cyberespace.

Mots Clés: Application, Droit économique et Commerce en ligne. Copyright @ 2020: This is an open-access article distributed under the terms of the Creative Commons Attribution license which permits unrestricted
use, distribution, and reproduction in any medium for non-commercial use (NonCommercial, or CC-BY-NC) provided the original author and source are credited.

\section{Problematique}

Enclin à la mondialisation, les Etats, développés et sous-développés se bousculent apports en mains pour effectivement prendre part au grand rendezvous du donner et du recevoir. L'ampleur de l'artefact entraîne avec celui-ci bien de faits bouleversant et novateur. Le dernier en date peut s'avérer sans conteste l'avènement des nouvelles technologies et de l'internet, qui s'inscrit dans le prolongement du processus de mondialisation du commerce. La multiplicité de contacts et de contrats transfrontaliers de nature tant civile que commercial n'achève d'en convaincre.

Les auteurs sont légion à qualifier ce $21^{\mathrm{e}}$ siècle de l'ère du numérique qui se traduit par l'explosion des nouvelles technologies dont le reflet s'avère la conquête de la quasi-totalité de la vie de l'humain à tel enseigne que celui qui possède la maîtrise de l'outil communicationnel et informatique serait le lettré du futur. L'on s'aperçoit donc qu'internet n'est pas un phénomène de mode sujet à passer tel le temps emporte le vent. C'est une réelle sphère d'activité à part entière au rôle vraisemblablement capital dans l'économie du $21^{\mathrm{e}}$ siècle où différents secteurs et acteurs économiques se côtoient en vertu de la plénitude du principe de libre entreprise.
Il ressort de l'irruption de l'internet, une réalité de développement sans précédent des transactions par ce canal modifiant considérablement la perspective traditionnelle du commerce classique en le plongeant dans un mode électronique inhabituel, virtuel et ouvert. Cette ouverture sur le monde paraît profitable au consommateur qui identifie plus rapidement la totalité de l'offre disponible à travers le monde et sur laquelle, il peut opérer des choix conséquents où qu'il soit. Parallèlement, les opérateurs économiques présents sur internet ont l'opportunité de toucher un plus grand nombre de consommateurs et sont déchargés de certaines charges tels les frais de magasin, de dépôt de stock, et d'autres frais tels la publicité, l'impôt, etc.

La rapidité, la simplicité, le moindre coût, les libertés et autres vertus qui jonchent le web et conduisent sans détour à offrir des prix plus compétitifs dont le mode de paiement est sécurisé comme il est simplifié. Internet reste dès lors ce cadre qui donne plus de chance à croiser des nouveaux débouchés.

Malgré, les avancées spectaculaires et les résultats jusque-là très prometteurs réalisés par le commerce via internet, la définition des rapports juridiques nés en son sein n'a pas l'air aisé et pose 
désormais le problème de son organisation sur le plan juridique. D'ailleurs, la querelle entre la position américaine laissant les liens juridiques ainsi créés à la merci de la volonté des utilisateurs c'est-à-dire voués à la diversité des lois du monde et la position européenne qui prône l'harmonisation des dispositifs législatifs et réglementaires dans le sens des nouveaux problèmes suscités par internet ne fait que réconforter le malaise. Pourtant en dépit de ces questions qui divisent et unifient dans la pluralité la doctrine, le droit congolais sombre encore dans la léthargie; c'est donc dans ce contexte d'impasse juridique qu'il se voit interpellé.

Lui faudrait-il circonscrire juridiquement un marché prématurément émergent, dont les standards de comportement ne sont pas encore stabilisés et dont les pratiques sont encore balbutiantes ou ne serait-il pas louable d'analyser les domaines ou les lois actuelles s'appliquent déjà, quitte à adapter, (...), certaines dispositions aux spécificités du commerce.

C'est à ces "fractures" juridiques que s'efforcera de répondre le présent article, et aura comme objectif essentiel de soulever l'impact des dispositions commerciales et économiques classiques en vigueur en République Démocratique du Congo sur le commerce en ligne. Et de vérifier s'il est possible de prétendre à la réalisation d'un consentement électronique libre et éclairé.

\section{HYPOTHESE}

Internet ouvert à tous les coins et recoins du monde se retrouve soumis aux lois quels qu'en soient l'ordre juridique. De ce fait, nous conviendrons qu'internet n'est pas à l'ombre du droit d'autant plus qu'il est soumis à une pléthore des règles juridiques parfois distinctes mais, qui de concert avec certaines conventions sont promptes à circonscrire l'univers virtuel qu'il incarne.

De parfait accord avec le passage précédent, la législation congolaise par quelques-unes de ses dispositions et les conventions dûment ratifiées sont à même de réguler le commerce, les obligations et les litiges afférents au commerce nés sur internet rien qu'en se fiant au schéma classique du droit, sans prétendre suffire néanmoins à la totalité d'épineuses questions inhérentes à internet.

\section{Interet de l'article}

Fasciné par l'abondante complexité juridique qui fait l'arrière-plan de l'internet, nous nous sommes engagés à creuser le fond de la question à la lumière de la législation congolaise en matière économique afin d'en ressortir les originalités et cerner au mieux la question sur le plan national.

Internet se révèle certes une réelle sphère d'activité à caractère civil et commercial sur le marché planétaire, libre, ouvert et direct qu'il propose; corrélativement, c'est le vecteur d'un autre genre de risques et même de délit qui appelle la sécurité dans le réseau par une réglementation et une jurisprudence particulière. Il devient donc nécessaire d'examiner le degré d'efficacité de la législation commerciale congolaise en vigueur dans l'espoir de planifier un cadre fiable et sécurisé profitable aux acteurs commerciaux congolais qui déjà achètent et vendent en ligne.

L'intérêt de l'article se justifie aussi dans la perspective d'adoption indispensable des nouveaux modèles commerciaux qu'impose sur le plan mondial les techniques de l'information et de la communication (TIC). Les TIC peuvent être des moyens efficaces de commercialisation de l'offre et d'accroissement de la clientèle (Conférence des Nations Unies sur le commerce et le développement, du 30 novembre au 2 décembre 2005 sur les TIC et le tourisme dans une optique de développement, p.3).

En tant que tel, elles permettent à un pays en l'occurrence la République Démocratique du Congo d'être plus autonome dans sa tâche de se trouver les débouchés nécessaires à son essor.

Ceci conduirait à ce que les entreprises d'un tel pays accèdent directement au marché international, traitent directement avec les consommateurs et distributeurs étrangers et accroissent leurs compétitivités.

Nous tenons donc à remettre à tous les praticiens du droit congolais, le sujet de nos recherches afin qu'avec vous, ce problème qui n'est pas encore à la une de nos préoccupations juridiques puisse préalablement trouver des pistes de solution pour qu'au temps convenu, nous brandissions le droit adéquat.

\section{Cadre methodologique}

L'idée centrale que nous proposons de développer, à cet égard, consiste à vérifier si les théories classiques du droit commercial, économique, fiscal et civil sont encore viables à la régulation du commerce qui cette fois emprunte les voies de l'internet.

La démarche que nous proposons d'adopter pour approcher la problématique de notre article est : de procéder à une approche exégétique. Autrement dit, de comparer les fondamentaux juridiques du commerce en ligne en ce qui concerne les principes de base, la présentation du produit ou du service, le processus de formation des contrats électroniques, le mode de paiement électronique et la fiscalité y afférente au regard de la législation en matière économique en République Démocratique du Congo.

Outre cette méthode, nous avons plus recouru à la technique documentaire, aux méthodes fonctionnaliste et dialectique. La première nous a 
guidés dans l'étude du fonctionnement du système juridique congolais relatif au commerce et à son exercice en République Démocratique du Congo. Quant à la deuxième, du fait qu'elle étudie l'évolution des faits sociaux en les mettant en opposition critique pour élaborer des règles (juridiques...) en vue d'un meilleur rapport social, s'est affirmé ainsi comme étant incontournable tout au long du travail.

\section{Delimitation de l'article}

La présente étude est loin sans faux une analyse intégrale de la diversité du réel à appréhender sur le commerce en ligne. Pour la petite illustration, nous ne parlerons nullement d'internet comme nouveau besoin de consommation au sein de la population même s'il présente un intérêt indéniable et entraîne l'essor de la vente d'ordinateur et des logiciels. Nous n'examinerons pas non plus les questions de droit pénal, de la responsabilité des fournisseurs d'accès et des hébergeurs liées à l'utilisation illicite de l'internet, comme d'un bon nombre de questions. Il s'agira juste de déterminer les axes prioritaires du web commercial permettant aux corps des règles éparses du droit commercial ou économique de s'appliquer sinon de s'y référer eu égard du droit international privé dans le règlement des différends extra frontaliers.

\section{Considerations d'ordre general sur l'internet}

Comprendre une notion peut résulter de la connaissance générale des concepts qui l'entourent. Sur ce, nous avons estimé opportun définir les notions clés et préciser le cadre de notre mémoire pour mieux évoluer dans la compréhension de bon nombre de question qui sont envisagées.

En effet, il est indispensable au juriste de bien cerner le fonctionnement du réseau internet puis subséquemment, du commerce en ligne pour en tirer les conséquences juridiques, en analysant les problèmes et le cas échéant, les résoudre. La règle de droit ne saurait être pertinente si elle méconnaît la réalité technique Rosalie C, 1999:120.

Internet pur phénomène social s'avère incontournable dans sa configuration actuelle et les atouts qu'il regorge. D'ici peu, il deviendra à en croire certains sondages, le média par excellence déjà qu'il associe la télévision, la radio, le journal, la photographie, les bibliothèques et autres.

Pourtant, le cyberespace n'est jamais que le reflet de la société "réelle" avec ses valeurs et ses manquements. Celui-ci pourtant qualifier de virtuel n'est en fait, que le miroir de ce qui se passe dans le monde reel[7].

L'idée de révolution qui l'accompagne souvent n'est due qu'à l'espace de communication large et dense encadré par la liberté de jouissance et par la liberté d'expression presque sans censure qu'il incarne, sans pour autant méconnaître les libertés intangibles.

Par réseau ouvert, l'on entend l'absence d'un "propriétaire" à proprement parlé sujet à en définir les conditions juridiques nécessaire à l'usage. En dépit de ce fait, des nombreux projets juridiques tendant à donner à l'internet un cadre juridique commun à tous les utilisateurs éparpillés dans le monde sont en cours d'élaboration ou sont déjà débattus.

L'ouverture mondiale du réseau des réseaux laisse entendre, sans ambages, l'absence des frontières facilitée par la virtualisation du monde matériel malgré la pratique des noms de domaines nationaux qui tentent de transposer les notions de territoire terrestre, maritime ni aérien au concept de l'internet aux fins d'en déterminer la portée ou le champ d'action. L'absence des frontières est ainsi, assortie de la prérogative de jouissance des mêmes droits en principe dans le chef des utilisateurs du monde entier.

Les obligations sont parfois distinctes selon les motivations ou la politique d'un pays ou d'une communauté régionale, comme il sera vu au fur et à mesure que nous développerons certains autres paramètres du champ d'application.

\section{Du commerce en ligne au regard de la legislation en matiere economique en r.d.c. $L$ 'exercice du commerce en droit congolais}

L'action législative fait parti de l'arsenal des mesures destinées à améliorer la sécurité des transactions commerciales en générale et de celles passées en ligne en particulier. Les deux ordonnances présidentielles:

$\cdot \mathrm{N}^{\circ} 87-242$ du 23 juillet 1987 portant création du service présidentiel d'études;

$\cdot \mathrm{N}^{\circ} \quad 87-243$ du 23 juillet 1987 portant réglementation de l'activité informatique en République du zaïre, régissant le domaine de l'informatique ne font pas allusion à l'activité commerciale qui aujourd'hui emprunte les voies de l'internet.

\section{Les conditions d'exercice de la profession commerciale \\ En accord avec Emile ARRIGHI de CASANOVA, les actes professionnels d'un} commerçant ou d'un industriel sont, quel que soit sa branche d'activité (vente, transport, titre de paiement) innombrables, les uns et les autres, cependant ont besoin d'un environnement juridique assurant la sécurité de leur accomplissement dans un contexte social determine kumbu ki ngimbi, 2004:14.

Dans la même voie, l'accès à la profession commerciale en dépit de ce que MASAMBA MAKELA qualifie de "sacro-saint" principe de la 
liberté du commerce et de l'industrie, n'est pas véritablement libre. Les conditions et exceptions liées à la personne désireuse de pratiquer le commerce et à l'accomplissement même des actes réputés commerciaux par la loi constituent un préalable incontournable et sine qua none que nous analysons dans cette partie.

\section{Principe de la liberté commerciale}

Timidement consacré par notre constitution, l'accès aux activités commerciales est libre sous réserve toutefois du respect des textes légaux et réglementaires en la matière. Principe à valeur constitutionnelle, l'initiative privée (art.35 de la constitution de la $3^{\mathrm{e}}$ République) implique que toute personne physique ou morale puisse s'établir dans une activité commerciale sans autorisation préalable de l'autorité publique. Exception faite aux étrangers dont certains secteurs tel le petit commerce et autres sont interdits.

\section{Fondement du principe}

Au Congo, l'accès aux activités commerciales est libre sous réserve toutes fois des textes légaux et réglementaires en la matière.

L'article 35 de la constitution de la troisième république de la R.D.C. précise que : «l'Etat garantit le droit à l'initiative privée tant aux nationaux qu'aux étrangers. Il encourage l'exercice du petit commerce, de l'art et de l'artisanat par les congolais et veille à la protection et à la promotion de l'expertise et des compétences nationales. La loi fixe les modalités de ce droit».

Il en vient que ce principe est et reste la règle fondamentale pour l'exercice du commerce au Congo aussi bien par les nationaux que les étrangers. N'empêche par contre que le législateur organise son cadre d'exercice pratique en indiquant objectivement et subjectivement les différents cas de limitation et les conditions d'exercice.

La loi $n^{\circ} 73 / 009$ du 05 janvier 1973, en son article 5 exclut pour ce faire l'étranger de l'exercice du commerce d'importation, d'exportation, de transit de gros, de demi-gros, de détail, etc.

\section{Contenu du principe de libre entreprise}

Ce principe traduit une double réalité dans la mesure où il exclut le monopôle en garantissant d'une part à chaque citoyen le droit de s'adonner à l'exercice de toute industrie ou négoce de son gré et parallèlement, il confère à chaque commerçant le droit de concurrencer l'autre dans les limites de la loi. Ainsi, le dit KUMBU KI NGIMBI, la libre concurrence devient le corollaire obligé de la liberté du commerce et de l'industrie kumbu ki ngimbi.
Ce principe fort d'attribut, ouvre l'exercice du commerce ou industrie sur tous les biens qui sont dans le commerce, au seul choix du commerçant (national ou étranger) qui décide comme il l'entend soit de donner naissance à une activité commerciale, soit d'assurer la continuité d'une autre exploitation déjà existante. Les garanties de contracter les relations d'affaires avec les personnes de son gré, de s'établir où il estime mieux sur toute l'étendue de la République Démocratique Congo, de débuter son commerce et d'en mettre fin quand il le juge nécessaire et opportun, s'avèrent intactes, libres et respectées.

Paradoxalement, la libre concurrence n'est pas consacrée dans la constitution, mais est reconnue à chaque commerçant comme les moyens suffisants et honnêtes de s'accaparer de la clientèle de son concurrent, censée n'appartenir à personne. Il a été prouvé que pratiquée dans le strict respect de la loi et de la capacité des intérêts en jeu, l'asphyxie de la libre concurrence reste licite quand bien qu'il est consacré des monopoles légaux à certaines entreprises publics (SNEL, REGIDESO,...). Une autre limitation licite de la libre concurrence est celle de restreindre le principe à une sphère de personnes réunissant les mêmes conditions d'accès à savoir la tenue de certains diplômes spécifiques (pharmaciens, etc.).

D'emblée, il sied de souligner que le principe de liberté commerciale et d'entreprise subit l'influence des mutations profondes qui ont succédées à l'indépendance, dans la politique et la forme du gouvernement.

\section{Etude sur la viabilite des fondamentaux de la fiscalite au regards du commerce electronique}

Internet est un réseau transnational, par nature indifférente aux frontières qui procède à l'encontre du pouvoir d'imposer avant tout lié à la souveraineté nationale.

L'espace virtuel qu'est l'internet, faute de matérialité, rabroue les bases d'identification précise des parties en cause, ainsi que des opérations réalisées. Enfin, la fiscalité s'appuie souvent sur les intermédiaires pour l'exercice et le contrôle des prélèvements fiscaux. Or, l'Internet crée une "désintermédiation " rendant possible l'élimination de tout type d'intermédiaire.

En effet, les opérations effectuées sur le réseau n'entrent dans le champ d'application que de certains impôts.

Concernant l'impôt sur le revenu, les opérations réalisées sur ou via l'Internet peuvent générer des bénéfices industriels et commerciaux et des bénéfices non commerciaux. De plus, des opérations boursières peuvent être effectuées par le biais du réseau, au-delà des frontières nationales. Les revenus qui en résultent sont imposables dans la catégorie des revenus 
de capitaux mobiliers (BENETEAU J., "La fiscalité de l'Internet",

www.financespubliques.com/jp/fiscinter.html consulté le 12.02.2020).

Les bénéfices des sociétés peuvent également résulter d'opérations réalisées sur l'Internet. Ils seront soumis à l'impôt sur les sociétés.

La taxe sur la valeur ajoutée peut aussi s'appliquer aux livraisons de biens et prestations de services effectuées sur ou via l'Internet. Les livraisons de biens en provenance d'Etats tiers pourront enfin donner lieu à l'application de droits de douane.

Si ces impôts sont applicables aux opérations effectuées sur l'Internet, ils ne peuvent concerner que certaines composantes du réseau. En effet, les diverses fonctionnalités de l'Internet ne permettent pas dans leur ensemble de générer des revenus imposables. La question est de déterminer dans quelle mesure il est possible de concilier les spécificités du réseau qui entraînent des difficultés d'application des règles fiscales traditionnelles avec l'application des règles fiscales concernées.

\section{Les difficultés d'application des règles fiscales traditionnelles à l'internet}

Il n'existe actuellement, au Congo, aucune règle fiscale propre à l'Internet en général et au commerce électronique en particulier. Cependant la tendance générale est de faire fonctionner l'espace virtuel sur base des principes de neutralité fiscale, de sécurité juridique et de simplicité.

\section{En effet, la neutralité fiscale n'a pas de valeur} juridique, son opposition farouche contre l'interventionnisme écarte la création d'un nouvel impôt spécifique aux utilisateurs de l'internet. Elle s'entend alors de la recherche des " moyens à mettre en œuvre pour permettre à un producteur ou à un distributeur de richesses ou de services de choisir librement le cadre juridique ou économique de son entreprise sans être influencé, dans ce choix, par des considérations fiscales ".

Par ailleurs, la clarté, la prévisibilité et la transparence des obligations fiscales s'avèrent indispensables au moyen de la certitude juridique pour consacrer un cadre serein au commerce en ligne. La simplicité s'entend dans les outils adéquats pour assurer des systèmes d'imposition compatibles avec les pratiques commerciales, les plus simples et les plus accessibles.

Pour les opérations effectuées via l'Internet et qui soient génératrices de revenus, la position actuelle du Gouvernement français est de les imposer dans les conditions de droit commun.
La querelle de l'espace virtuel et de la territorialité de l'impôt

Pour l'application de la loi fiscale interne dans l'espace, la notion de territoire joue un rôle essentiel car le pouvoir d'imposer est une compétence exclusive de l'autorité souveraine dans l'État. Ainsi, dans les régimes démocratiques, seul le législateur peut créer, modifier ou supprimer un impôt. Cette notion ne saurait s'accorder avec l'internet qui par essence est libre, gratuit et ouvert sur la sphère planétaire.

En effet, l'espace virtuel que constitue l'Internet est de nature à remettre en cause les règles de compétence fiscale fondées sur la notion de territoire d'autant plus qu'il est un réseau de communication transnational non assujetti à aucune délimitation territoriale physique. Une certaine relativité tend à personnaliser l'imposition des opérations effectuées sur le réseau selon qu'elles sont toujours le fait, directement ou indirectement, d'une personne physique ou morale.

Les règles de domiciliation fiscale de ces personnes vont alors s'appliquer et l'utilisation de l'Internet sera sans conséquence sur la détermination du lieu d'imposition des opérations effectuées.

Il en va ainsi, notamment, de l'impôt sur le revenu intimement lié à la notion du domicile fiscal.

Le principe de la territorialité de l'impôt subira un sérieux affront lorsqu'une personne physique effectue à titre principal des opérations imposables sur le réseau. L'incertitude se présente lorsqu'il faut déterminer le centre des intérêts économiques de la personne dans la mesure où la majeure partie de ses revenus provient d'opérations effectuées sur le réseau.

Les cabinets d'avocats en ligne offrant des services d'assistance et de conseils juridiques sont très concernés par cette réalité.

De l'efficacite partielle et insuffisante du regime
juridique congolais face aux transactions en ligne
Eu égard à l'ampleur des activités de consommation ayant lieu sur internet, il devient judicieux d'examiner le degré d'efficacité de la législation congolaise en vigueur quant à la protection des intérêts des cyberconsommateurs(Lire OWENGA ODIMBA E.L.,"La protection des cyberconsommateurs en droit congolais", lex

Electronica, $\quad$ vol.8 $\quad \mathrm{n}^{\circ} 1$

2002 ,

http://www.lexelectronica.org/articles/V8-

1/owenga.html, consulté le 12.02.2020).

L'extranéité caractéristique d'internet dit tout des lois susceptibles de régir une situation contractuelle à caractère internationale de même que les règles juridiques nationales de Droit International Privé qui peuvent être nombreuses, variées et contradictoires. 
Ainsi, la diversité des ordres juridiques est facteur de conflit dans la mesure où un contrat en conformité avec tel système de droit peut être prohibé dans tel autre.

En droit congolais, la forme de la convention est régie par la lex loci actus (loi du lieu de l'acte) tel que consacré par l'article 9 de la convention de Rome du 19 avril 1980 sur la loi applicable aux obligations contractuelles, tandis que la forme est régie par l'autonomie de la volonté des parties.

Les règles $\mathrm{du}$ droit international privé connaissent d'importantes limitations surtout quand il faut les envisager dans le cyberespace:

En effet, L'article 11 alinéa 2 du code civil congolais livre premier veut que: «Sauf intention contraire des parties, les conventions soient régies, quant à leur substance, à leurs effets et à leur preuve, par la loi du lieu où elles ont été conclues ». Le lieu de la signature de l'acte parait inapproprié à l'internet d'autant plus que celui-ci est dominé par les contrats entre absents c'est-à-dire signés à distance. La distance est aussi pour l'internet synonyme d'absence de territoire matériel, ce qui explique la difficulté de connaître le lieu exact de conclusion de la convention et par la même de déterminer quelles sont les conditions de formalisme qui doivent être respectées.

L'article 9-2 de la convention de Rome semble apporter de la lumière au problème du lieu de l'acte selon que : « un contrat est conclu entre des personnes qui se trouvent dans des pays différents, est valable quant à la forme s'il satisfait aux conditions de forme de la loi d'un de ces deux pays ».

Néanmoins, il s'écarte de la position congolaise qui retient le lieu de signature de l'acte pour déterminer le formalisme. Les parties peuvent y voir une restriction de la liberté.

Par ailleurs, l'autonomie de la volonté des parties régissant le fond, pourra se trouver en conflit avec celle des Etats dans la mesure où elle ne serait pas reconnue dans tel ou tel Etat ou qu'elle serait écartée au profit des domaines pour lesquels les dispositions de leur droit sont impératives (lois réelles, lois de police). Sur internet, l'autonomie de la volonté débouche à la lex mercatoria tel que dégagée par BERTOLD GOLDMAN. Ceci consiste à rédiger une clause sans références particulières à la conclusion d'un contrat en ligne.

Par exemple: "les dispositions du présent contrat devront être interprétées selon les principes généraux de droit"; "Toutes difficultés devront être réglées en raison et en équité"...
L'article 129 de la loi portant Organisation Fonctionnement et Compétencedes Juridictions de l'ordre judiciaire dispose: «(...), l'action peut être portée devant le juge du lieu dans lequel l'obligation est née ou dans lequel elle doit être exécutée ». Nous nous apercevons d'emblée, que non seulement les textes sus évoqués ont été élaborés dans la parfaite ignorance des nouvelles technologies qui n'implique pas par conséquent leur obsolescence, ils sont butés à des sérieux écueils.

Le dilemme à indiquer le lieu de la conclusion d'un contrat en ligne fait appel à la difficulté du tribunal compétent et l'ensemble débouche à l'inefficacité de la protection légale du consommateur congolais.

La doctrine internationale nous enseigne qu'aux Etats-Unis par exemple c'est la provenance de la caractéristique principale du contrat en l'occurrence, le lieu du vendeur qui remporte le bénéfice de la loi applicable. Pour les législateurs européen et québécois, le contrat est présumé conclu à l'adresse du consommateur.

De ce fait, le consommateur n'est pas confronté à toutes les lois du monde que fait intervenir internet, seule la loi de son pays suffit. Néanmoins, l'article 11 al.2 a été édicté sous réserve au cas où les parties se conviennent de subir la rigueur d'une autre loi, le contrat requiert parfaitement sa validité.

D'où la loi congolaise est enclin à la conclusion des conventions déterminant la loi applicable mais à la condition que ces lois ne soient pas contraires à la législation en vigueur en République Démocratique Congo. C'est l'article 15 du code civil congolais livre premier qui l'illustre en ces termes: «Les lois, les jugements de pays étrangers, les conventions et dispositions privées ne peuvent en aucun cas avoir d'effet dans l'EIC en ce qu'ils ont de contraire au droit public de cet Etat ou à celles des lois qui ont en vue l'intérêt social ou la morale publique ».

\section{Inefficacite dans la formation du contrat}

La formation du contrat fait intervenir:

\section{Le devoir d'information}

La tendance législative Internationale est que l'utilisation des nouvelles technologies ne nuisent pas à l'information claire, complète et sans ambiguïté sur l'objet du contrat dans le chef du consommateur.

Néanmoins, la complexité de la toile est une aubaine pour certains détracteurs qui peuvent déformer les informations utiles à la présentation soit du produit, soit de l'entreprise pour soit s'attirer la clientèle de tel concurrent, soit pour une autre raison. Ce qui n'est pas sans conséquence d'ailleurs, le rendement du marché en souffre, la clientèle et les règles du jeu équitable. 
La législation congolaise frappait de mutisme absolu en cette matière, aborde indirectement et sans l'intention de s'y référer en prônant la connaissance parfaite des enjeux afin d'effectuer un choix éclairé.

Elle peut en effet brandir l'article 38 du code civil congolais livre III qui confère la responsabilité des vices cachés au vendeur. L'article 279 du même code énonce que : «le vendeur est tenu d'expliquer clairement ce à quoi il s'engage. Tout pacte obscur et ambigu s'interprète contre le vendeur $»$.

\section{L'offre}

Malgré l'article 270 du code civil congolais livre III: « La promesse de vente vaut vente, lorsqu'il y a consentement réciproque des deux parties sur la chose et le prix »; l'offre n'est pas strictement réglementée en droit congolais. La législation congolaise n'aborde pas le problème de l'acceptation de l'offre de contracter néanmoins les parties aux contrats doivent s'en remettre aux règles de preuve du droit commun pour établir l'acceptation.

L'article 9 du décret-loi du 20 mars 1961 sur le prix tel que libellé : «Il est interdit à tout commerçant, industriel, producteur agricole et artisan:

$1^{\circ}$ de refuser à tout commerçant de satisfaire, dans la mesure de ses possibilités aux demandes des acheteurs de produits ou aux demandes de prestation de services lorsque ces demandes ne présentent aucun caractère anormal et qu'elles émanent de demandeurs de bonne foi ;

$2^{\circ}$ de subordonner la vente d'un produit ou la prestation d'un service quelconque soit à l'achat concomitant d'autres produits, soit à l'achat d'une quantité imposée, soit à la prestation d'un autre service », remplirait mieux sa mission sur le monde physique. Transposée sur internet, cet article s'avère inefficace puisque le problème d'identification sur internet et celui de la territorialité des transactions et des sujets viendraient à s'y opposer, en sus le réseau étant ouvert la crainte n'est pas fondée aussi longtemps qu'il y aura une quantité infini des produits mis en ligne.

Elle ne contient aucune disposition autorisant un consommateur à retourner au vendeur un bien acheté à distance quand il considère s'être trompée, ne sent plus l'utilité du produit ou pense que le prix ne correspond pas à ses moyens.

Cette carence juridique n'offre pas la possibilité de conférer un traitement particulier aux contrats adaptés aux réalités du cyberespace.

\section{La publicité}

Nous n'exposerons pas à nouveau la notion sur la publicité ou sur le devoir d'information déjà entretenues. Notons seulement qu'il n'existe pas en droit congolais de réglementation sur la publicité en dehors de celle sur les produits pharmaceutiques pour le fond et de pour la forme. Il s'agit de l'ordonnance $\mathrm{n}^{\circ} 72 / 6 \mathrm{du}$ 2 janvier 1958 relative à la publicité en matière pharmaceutique et vente, cession ou délivrance de médicaments en dehors des officines.

Ainsi, en ce qui concerne l'appréciation du contenu d'une publicité relative au secteur pharmaceutique et faite à partir du réseau internet, l'ordonnance $\mathrm{n}^{\circ} 72 / 6$ du 2 janvier 1958 peut s'avérer utile pour les juges, surtout lorsqu'il n'existe aucun élément d'extranéité. Cependant, les problèmes de territorialité et d'indentification inhérents à l'internet rendent moins aisé l'application de l'ordonnance $n^{\circ} 72 / 6$ du 2 janvier 1958 lorsqu'il faut apprécier une publicité dont la source n'est pas congolaise. Une publicité sur internet sur un produit qui irait à l'encontre des prescrits du législateur congolais a du mal à être sanctionnée par celle-ci surtout que sa source est étrangère et que son public cible n'est pas congolais.

Sur le plan de la forme, «La publicité faite à partir des sites qui font la publicité ne reprend pas la mention "publicité" dans leurs messages publicitaires de sorte qu'il paraît encombrant d'imposer cette règle aux sites web ». De même, « un grand nombre de sites révèlent déjà leur mission de diffuseurs de données publicitaires par la nature des messages qu'on y trouve ».

A en croire l'article 27 de l'ordonnance législative $\mathrm{n}^{\circ} 254 /$ Téléc du 23 août 1940 sur les télécommunications qui interdit l'usage des voies de télécommunication pour des publicités d'affaires, la publicité faite par courrier électronique est interdite si elle se situe en matière commerciale.

En dépit de l'absence d'un texte de portée générale sur le fond, il est permis de soutenir que toute publicité dont le contenu est mensonger ou trompeur ne peut être admise. La concurrence déloyale sanctionne certains actes considérés comme contraires aux usages honnêtes en matière commerciale. «Le congolais n'a donc que peu de pouvoir pour prohiber la vente du produit concerné par la publicité surtout quand la livraison est faite par téléchargement ».

\section{Les prix}

«L'obligation de l'affichage des prix des biens peut certes s'appliquer aux site internet, mais elle soulève des interrogations. Comment les agents économiques à qui l'article 4 du Décret-loi du 20 mars 1961 tel que modifié et complétée en a donné le pouvoir vont-ils procéder aux enquêtes relatives à la fixation du prix ? Comment identifierons-ils les sites assujettis à la législation congolaise ? En fait, certains des pouvoirs reconnus à ces agents semblent difficiles à exercer sur le réseau Internet. C'est le cas, notamment 
du pouvoir de procéder aux visites d'établissements commerciaux $»$.

L'obligation de délivrance de la facture pour toute vente au détail et pour toute prestation de service ne dépassant pas 500 francs congolais semble être surannée sur internet où il est difficile d'assujettir les sites et les clients congolais à «pareille exigence, à moins de permettre la facture numérique dont la notion, les caractéristiques et les modalités méritent d'être préalablement définis par un loi ».

\section{Inefficacite des principes d'imposition a la source et au lieu de residence}

Sauf abus, jusqu'à ce jour le commerce électronique n'est doté d'aucun régime fiscal; en matière de procédure, «il est régi par les dispositions générales incorporant un agrégat de régimes particuliers (ceux des télécommunications, des produits informatiques et de la vente à distance) ».La jurisprudence quasi inexistante, l'Internet Tax FreedomAct américain, n'est qu'un moratoire qui ne propose d'ailleurs aucun régime fiscal.

L'imposition au lieu de résidence s'avère simple et naturelle dans la mesure où elle s'applique à des contribuables dotés d'attache économique, personnelles, sociales et professionnelles les liant à l'Etat de résidence au point que celui est pourvu des moyens de contrôles sur ces contribuables et sur qui il peut s'informer plus aisément. Le droit fiscal congolais excelle tellement dans ce domaine qu'il lui arrive d'écarter l'imposition à la source, sa transposition sur internet n'est pas certaine. La résidence étant le critère d'imposition ultime, la dématérialisation n'ayant pas encore atteint l'individu, la référence au domicile fiscal apparaît comme un gage de sécurité juridique, à l'inverse au siège des personnes morales, qui peut être aisément manipulé.

Les moyens modernes pourraient rendre difficile la détermination du lieu de résidence des entreprises, ces mêmes entreprises peuvent profiter de l'immatérialité pour localiser artificiellement leur résidence dans des territoires à fiscalité privilégiée. En droit congolais, déjà que le législateur lui-même par le biais de l'ordonnance ${ }^{\circ} 69-009$ du 10 février 1969 relatives aux impôts sur les revenus, «renonce à imposer tous les revenus des sources étrangères au profit de l'aspect réel de la territorialité de l'impôt. L'article $1^{\text {er }}$ de l'ordonnance précitée est explicite à ce sujet lorsqu'il déclare qu'il est établi un impôt mobilier sur les revenus des capitaux mobiliers investis en République Démocratique du Congo.

Il en résulte que les dividendes, les intérêts et les redevances produits par les capitaux mobiliers investis à l'étranger sont exonérés de toute imposition en République Démocratique du Congo ».
Par contre, l'imposition à la source fondée sur l'équité fait intervenir une mise en œuvre difficile qui tient du non résidence des contribuables, mais qui opère par voie de convention Internationale.

La règle d'imposition à la source pouvant se traduire de plusieurs manières : imposition au lieu de production (pour les revenus d'activités commerciales via l'établissement stable), au lieu du domicile du siège social du débiteur (intérêts, dividendes) ou au lieu d'exécution des services ou du travail (pour les revenus d'activités non commerciales), la source du revenu dépend donc de la nature de celui-ci. Néanmoins quelle que soit la source du revenu, il n'est possible de collecter l'impôt pour le compte de l'Etat de la source que si le contribuable possède un établissement stable dans cet Etat ou si un intermédiaire chargé de prélever une retenue à la source, y est présent.

Cependant, le commerce électronique vient bouleverser ce schéma bien assis depuis des décennies en supprimant les intermédiaires habituelles et la nécessité d'une présence physique dans l'Etat de la source du revenu. «Ce qui rend difficile la détermination de la source du revenu, et donc la détermination de l'Etat dans lequel la retenue doit être effectuée, alors que la désintermédiation rend aléatoire sa perception $»$.

Une retenue à la source est généralement prévue par le droit interne ou le droit conventionnel concernant les revenus de non-résidents appartement à certaines catégories (dividendes, intérêts, salaires, redevances). Les législations internes prévoient aussi l'imposition dans le pays de la source des revenus non commerciaux. En effet, deux critères servent à déterminer la source des revenus:

- L'exécution matérielle, plus utilisé par les pays industrialisés ;

- Lieu d'utilisation, utilisé par les pays en voie de développement.

La question de déterminer la source du revenu dans le commerce électronique n'a eu pour réponse que l'impossibilité d'en déterminer la méthode quel que soit le critère de la source du revenu. La disparition des intermédiaires habituels (grossistes, détaillants, transporteurs, banques), objet de la dématérialisation et de l'interactivité sur internet empêche le recouvrement de l'impôt. En sus, l'utilisation de la carte de crédit tend à effacer à court terme le rôle traditionnel des banques, celui d'effectuer une retenue à la source pour certains revenus.

\section{CONCLUSION}

Au terme de notre dissertation qui a portée sur : « les modalités d'applications des règles du droit économiques congolais dans la pratique du commerce en ligne », il sied de retenir qu'il existe des lois à 
retrouver ça et là dans divers codes congolais pouvant réguler tant soit peu le commerce en ligne.

Relevons a fortiori qu'il est une évidence opportune aujourd'hui de clamer la nécessité d'abord de consolidation et d'enrichissement des dispositions encadrant la pratique "normale" du commerce en République Démocratique du Congo avant d'envisager les prétentions d'harmonisation et d'adaptation aux nouvelles technologies. Mais à l'issue de divers manquements constatés le long de cette étude, et à l'instar de nombreuses législations dont souvent nous sommes héritiers des grandes lignes, il apparaît aujourd'hui nécessaire de prendre conscience de ce que seront les prochains marchés du consommateur congolais.

Prendre conscience pours UN juriste, peut être synonyme de prévoir la limitation d'abus afin de garantir la paisible jouissance d'un droit notamment le droit de consommation. Il n'est pas encore certes, un souci majeur pour le législateur congolais de circonscrire juridiquement un domaine qui est quasiment inexistant, sinon méconnu du consommateur congolais.

Mais, conscient de l'ampleur que prennent les débats à ce sujet, une anticipation législative de notre droit positif tomberait bien à propos.

\section{REFFERENCE}

\section{Textes réglementaires et législatifs}

1. Loi ${ }^{\circ} 73-009$ du 5 janvier 1973 sur le commerce in journal officiel de la république du Zaïre, $\mathrm{n}^{\circ} 5$, $1^{\mathrm{er}}$ mars 1973, P.280

2. Loi n ${ }^{\circ} 87-1-010$ du $1^{\text {er }}$ août 1987 portant Code de la famille in journal officiel de la_République de Zaïre, ${ }^{\circ}$ spécial, $1^{\mathrm{er}}$ août 1987

3. Loi $n^{\circ} 77-027$ du 17 novembre 1977 portant mesures générales de rétrocession des biens zaïrianisés ou radicalisés in journal officiel de la République du Zaïre, $\mathrm{n}^{\circ} 1,1^{\mathrm{er}}$ janvier $1978, \mathrm{P} .8$

4. Ordonnances $n^{\circ} 87-242$ et $n^{\circ} 87-243$ du 22 juillet 1987 portant respectivement création du service présidentiel d'études et réglementation de l'activité informatique en République du Zaïre in journal
Officiel de la République du Zaïre, $\mathrm{n}^{\circ} 15,1^{\mathrm{er}}$ août 1987, P. 21

5. Ordonnance-loi du 12 septembre 1983 relative à la police des étrangers in journal officiel_de la République du Zaïre, $\mathrm{n}^{\circ} 18,15$ septembre 1983,P.15

6. Ordonnance-loi $\mathrm{n}^{\circ} 66 / 260$ du 21 avril 1966 subordonnant à des garanties financières l'immatriculation au registre du commerce des étrangers, des sociétés étrangères et de certaines sociétés congolaises in moniteur congolais, P.243

7. Décret du 06 mars 1951 portant institution du registre de commerce in Bulletin Officiel, 1951, P.291

8. Décret du 02 août 1913 des commerçants et de la preuve des engagements commerciaux in Bulletin Officiel, 1913, P.775

9. Décret du 27 février 1887 portant sur les sociétés commerciales in Bulletin Officiel, 1887, P ; 24, nouvelle édition P.150

10. Décret-loi du 20 mars 1961 portant réglementation des prix in Moniteur Congolais, 1961, P.218

\section{Ouvrages}

1. Bensoussan, A. (2003). Informatique-TélécomsInternet : réglementation, contrats, fiscalité, communications électroniques, Paris, Francis Lefebvre

2. Breton, Ph. (2000). Le culte de l'internet une menace pours le lien social, éd. La découverte, Paris, Litec

3. Huet, F. (2000). La fiscalité du commerce électronique : impôt et territorialité, régime fiscal, pouvoir de l'administration fiscale, Paris, Litec,

4. Lamizet, B., E.T Silim, A. (1997). Dictionnaire encyclopédique des sciences de l'information et de la communication, Paris, Ellypses,

5. Lepage, A. (2002). Liberté et droits fondamentaux à l'épreuve de l'internet: droit de l'internaute, liberté d'expression sur internet, responsabilité, Paris, Litec,

6. Masamba, M.R. (1996). Droits des affaires : cadre juridique de la vie des affaires au Zaïre, Kinshasa, Cadicec

7. Sfez, L. (1993). Dictionnaire critique de la communication, Paris, PUF.

8. Thieffrit, P. (2002). Commerce électronique: Droit international et européen, Paris, Litec. 